\title{
Good practices in provision of nuclear safeguards and security training courses at the Integrated Support Center for Nuclear Nonproliferation and Nuclear Security
}

\author{
Naoki Kobayashi ${ }^{\text {a }}$, Kalman A. Robertson, Mitsuo Koizumi, and Yosuke Naoi \\ Japan Atomic Energy Agency (JAEA), Tokai-mura, Ibaraki, Japan
}

\begin{abstract}
More than five years have passed since the Integrated Support Center for Nuclear Nonproliferation and Nuclear Security (ISCN) was established under the Japan Atomic Energy Agency (JAEA) in December 2010 and started its activities, in response to the commitment of Japan at the Nuclear Security Summit in Washington D.C.. The ISCN has been vigorously involved in capacity building assistance on nuclear nonproliferation (safeguards) and nuclear security, mainly in the Asian region. It has provided 105 training courses to 2901 participants in total as of August 2016. The ISCN plays a major role in strengthening nuclear nonproliferation and nuclear security in the region, and this can be considered one of the great results of the Nuclear Security Summit process. The ISCN has cooperated with the US Department of Energy/National Nuclear Security Administration (DOE/NNSA) and Sandia National Laboratories (SNL) to establish a base of instructors, particularly for the Center's flagship two-week courses, the Regional Training Course on Physical Protection of Nuclear Material and Facilities (RTC on PP) and the Regional Training Course on State Systems of Accounting for and Control of Nuclear Material (RTC on SSAC). Furthermore, the ISCN has provided training courses for experts in Japan, making the best use of the Center's knowledge and experience of organizing international courses. The ISCN has also started joint synchronized training with the Joint Research Centre of the European Commission (EC JRC) on nuclear safeguards. This paper describes the good practices at the ISCN through its five years of activities, focusing on its progress in nuclear safeguards and nuclear security training.
\end{abstract}

\section{Introduction}

Within the fields of nuclear nonproliferation and nuclear security, the greatest need for capacity building is among states that are developing their infrastructures to support new or expanding nuclear energy industries, as is the case for several countries in Asia. During the 2010 Nuclear Security Summit in Washington DC, Japan committed to establishing a center of excellence for nuclear security to provide assistance with capacity building and technology development, primarily to states in Asia. The Integrated Support Center for Nuclear Nonproliferation and Nuclear Security (ISCN) was established within the Japan Atomic Energy Agency (JAEA) in December 2010. The JAEA has experience developing and operating nuclear technology in all steps of the nuclear fuel cycle and is well placed to assist states with capacity building in the fields of nuclear safeguards and nuclear security.

Over the last five years, the ISCN has conducted training activities focusing on the development of the knowledge, skills, and attitudes required to fulfill safeguards commitments and maintain effective national nuclear security regimes. This paper describes the good practices of the ISCN in the provision of nuclear security and safeguards training over the last five years. It explains how the ISCN has developed to fulfil the training needs created by recent changes to legal instruments

a e-mail: kobayashi.naoki44@jaea.go.jp and guidance documents on nuclear security, as well as the widespread adoption of the additional protocol to safeguards agreements. It discusses the ISCN's use of unique training tools and the Center's efforts to continuously improve its training programs. It then briefly outlines the ISCN's international/regional, bilateral, and domestic offerings, as well as its IAEA inspector training courses.

\section{Overview of the ISCN's training courses}

The ISCN conducts three types of courses: Nuclear Security Courses, Safeguards and State System of Accounting and Control (SSAC) Courses, and International Nonproliferation Framework Courses [1]. As of August 2016, the ISCN has conducted 105 training courses and trained a total of 2901 participants from 73 countries (including Japan) and three international organizations.

Many of the ISCN's courses are international/regional, allowing the Center's International Capacity-Building Support Office to assist many states with limited resources. The ISCN provides tailored bilateral dispatching courses where individual states request special assistance. The ISCN has also developed training programs in response to demand among Japan's regulatory/security authorities and operators. 


\section{Total 2901 participants in 105 courses (73 countries, 3 international organizations)}

course topic

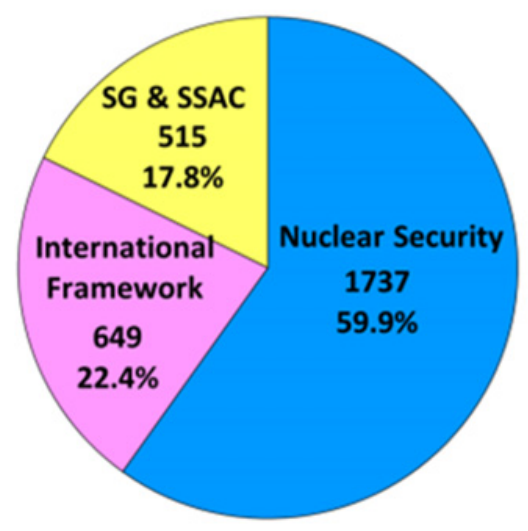

nationality

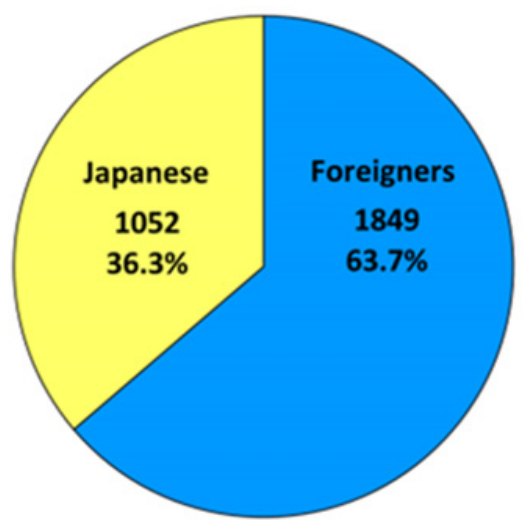

course style

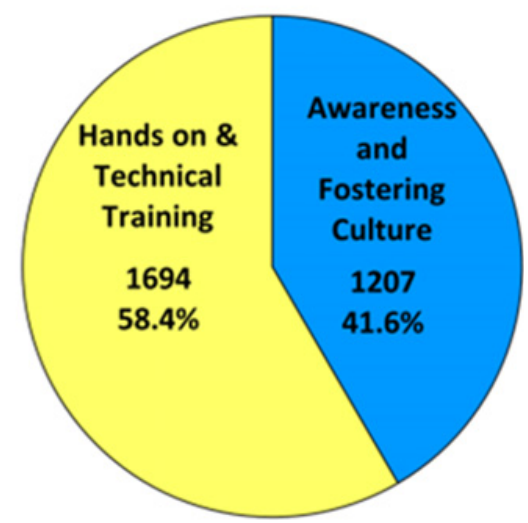

Figure 1. Activity results of the ISCN from 2011 to August 2016.

\section{The ISCN's development of training courses and cooperation with other training providers}

In order to analyze the training needs of potential recipients in the region, the JAEA began conducting training needs surveys in Southeast Asian states in 2010. The ISCN later expanded these missions to a number of countries in Central and South Asia, the Middle East and Eastern Europe. By the time the ISCN was established, the JAEA already had over ten years of experience offering training on safeguards but it did not have a complete course curriculum on physical protection. To help develop the ISCN's base of instructors for nuclear security, the ISCN partnered with the US Department of Energy/National Nuclear Security Administration (DOE/NNSA) and Sandia National Laboratories (SNL) to host a train-thetrainer (pilot) course on physical protection in August 2011. SNL provided lectures and materials based on its long-standing three-week International Training Course on the Physical Protection of Nuclear Material and Nuclear Facilities. The ISCN then began hosting its annual two-week Regional Training Courses on Physical Protection in cooperation with DOE/NNSA and SNL in October 2011.

Since 2011, the ISCN has progressively developed its course curricula, adding practical training tools and exercises, in response to feedback from national needs surveys and course participants [2]. The JAEA's Physical Protection Exercise Field (completed in 2012) allows participants to gain hands-on experience with real physical protection equipment in a realistic environment. Participants learn the functions of each component of a physical protection system and have the opportunity to act as Central Alarm Station officers, security guards, police officers, or intruders. The Field is also designed to facilitate realistic equipment performance assessment. Similarly, the ISCN incorporates real containment and surveillance equipment, including an EOSS seal and a DCM-C5 camera, into the hands-on component of the RTC on SSAC course.
The JAEA's Virtual Reality System allows participants to train using a detailed three-dimensional simulation of a realistic facility. Virtual reality allows access to all parts of the facility and allows participants to view detection zones (sensing areas and blind spots) for different configurations of physical protection equipment. It also supports simulations of security incidents. In 2016, the ISCN is also applying the Virtual Reality System to demonstrate nuclear material accounting for its RTC on SSAC. To supplement these realistic training environments, DOE/NNSA recently provided a Material Balance Area (MBA) kit to facilitate hands-on, simulated accounting exercises using mock-up bulk items [3].

The ISCN's two-week training courses include visits to Hiroshima or Nagasaki, incorporating the good practice of placing the gravity of the threat of nuclear proliferation in context. The horror of the atomic bombings serves as a stark reminder of the importance of controlling the use of nuclear materials and the visit is a valuable experience for practitioners in this field.

The ISCN has introduced country presentations into its courses to facilitate discussion of the specific experiences and capacity building needs of states in the region. Furthermore, the ISCN has supported Japan's chairmanship (2014 to present) of the AsiaPacific Safeguards Network, which has created additional opportunities to analyze the training needs of organizations and states [4]. In 2015, the ISCN conducted surveys on regional training needs and providers with the aim of assisting the APSN to optimize coordination of training programs.

As more training centers open, harmonization of training activities is becoming increasingly important. The ISCN discusses the scheduling of training activities and the harmonization of course contents with other members of the IAEA's International Network for Nuclear Security Training \& Support Centers, particularly the other Centers of Excellence in Asia [5].

The Joint Research Centre of the European Commission (EC JRC) and the ISCN often work together to provide courses. The Japan Atomic Energy Research 
Institute (JAERI, a predecessor of the JAEA) concluded an agreement with the European Atomic Energy Community in 1990 on cooperation in the field of safeguards research [6]. Since then the agreement has been expanded to incorporate capacity building in the areas of safeguards, security and non-proliferation, including coordination of technical support to third countries $[7,8]$. The ISCN and EC JRC have cooperated on curriculum development and exchanged lecturers for their courses.

\section{Regional training courses}

The ISCN and the EC JRC have cooperated on some of their international safeguards courses. The EC JRC arranged with the ISCN to conduct follow-up courses on non-destructive analysis for some of the participants to the ISCN's RTC on SSAC in 2015 and 2016. The EC JRC's follow-up courses use real nuclear material at the Ispra site to extend the skills of personnel from state regulatory authorities tasked with verifying operators' declarations using NDA techniques.

In addition to the ISCN's RTC on PP, the ISCN has taken advantage of its growing expertise to conduct or contribute to courses on nuclear forensics, nuclear security culture, measures against sabotage, computer security, insider threats, security of radioactive sources, and transport security. The ISCN has also been providing courses with emphasis on universalization of the IAEA's Nuclear Security Recommendations on Physical Protection of Nuclear Material and Nuclear Facilities (INFCIRC/225/Rev.5). The IAEA recognized the good practice of the ISCN and SNL in providing training to states on the practical implementation of the IAEA guidelines and requested that the ISCN and SNL transfer their workshop curriculum. The IAEA, the ISCN and SNL then worked together to establish an expanded five-day IAEA Regional Training Course on INFCIRC/225/Rev.5 in 2013.

\section{Dispatching courses}

The ISCN has also tailored training cooperation to appropriately match the needs of individual states that request the ISCN's assistance. The ISCN co-organized its first dispatching courses on additional protocol declarations and nuclear security with the Vietnam Agency for Radiation and Nuclear Safety in Hanoi in October 2011. Since then, the ISCN has conducted dispatching courses on safeguards or security in eight states. In 2013, the ISCN co-organized a workshop on border security monitoring and radiation assessment equipment with EC JRC and DOE/NNSA for Lithuania's new Center of Excellence in its Border Guard School. The EC JRC provided test reagent and a mobile detection system for hands-on exercises during this workshop.

The ISCN is also cooperating with the European Union Chemical, Biological, Radiological and Nuclear Risk Mitigation Centres of Excellence Initiative in this area. In October 2016, the ISCN will partner with the Initiative's Regional Secretariat for the Middle East to arrange a joint seminar in Amman, Jordan on Regional Cooperation to Enhance a Worldwide Nuclear Security Culture.

\section{IAEA inspector training}

Since 2011, the ISCN has also contributed to the enhancement of the effectiveness of safeguards implementation by providing an annual training course primarily for IAEA inspectors on safeguarding reprocessing facilities and activities. The ISCN has also helped to provide training to the IAEA in other areas, including the use of Digital Cherenkov Viewing Devices for spent fuel verification.

\section{Domestic courses}

The ISCN began providing training on physical protection for Japan's operators and regulators in 2012, after translating the course materials developed with SNL for the RTC on PP into Japanese and modifying them to match the specific requirements of Japan's physical protection regulations. The ISCN has also provided physical protection and emergency response training courses for security authorities that have requested its assistance, including the Chemical School of the Japan Ground Self-Defense Force, the Japan Coast Guard, and the National Police Agency. The total number of Japanese experts that have completed ISCN courses has surpassed 1000 .

Several operators in Japan have also requested the ISCN's assistance with raising awareness among their broader personnel of nuclear security threats and individuals' responsibilities. In response, the ISCN has introduced a Nuclear Security Culture lecture series for nuclear power plant (NPP) sites throughout Japan [9]. In 2015, the ISCN delivered the lectures at 13 NPP sites and 2300 NPP workers joined the lectures. This demonstrates that the ISCN has been recognized as an expert organization for nuclear security training support in Japan.

\section{Conclusion}

The ISCN was one of the first Centers of Excellence to arise from the Nuclear Security Summit process. The training courses at the ISCN assist states to develop the human resources necessary to meet their safeguards commitments and enhance nuclear security. The ISCN's training activities also promote understanding among individuals working in state regulatory authorities, government departments, nuclear facilities, and research institutes about how their responsibilities fit into the overall objective of nuclear nonproliferation. The ISCN has built up a central repository of knowledge and instructors in cooperation with other training providers, allowing the ISCN to expand its course offerings. The ISCN will continue working closely with the IAEA and other training providers with a view to enhancing the effectiveness and availability of training.

\section{References}

[1] R. Matsuzawa, "From Asia to the World: Evolvement of ISCN/JAEA as a Training Center for Nuclear Nonproliferation and Nuclear Security" 1540 Compass 10 (Spring 2016) 18-21

[2] M. Hirai, Y. Naoi, N. Kobayashi, and Y. Nakamura, "Providing Effective Human Capacity Programs for Asian Countries: Based on the Feedback from 
Participants" (Paper presented to Institute of Nuclear Materials Management $57^{\text {th }}$ Annual Conference, Atlanta, July 27, 2016)

[3] M. Schanfein et al., "Teaching the Principles of Safeguards Inventory Inspections: Material Balance Area Kit" (Paper presented to Institute of Nuclear Materials Management $57^{\text {th }}$ Annual Conference, Atlanta, July 26, 2016)

[4] Y. Okumura, “The Working Group 2: SG Capacity Building Action Plan Proposal" (Presentation to $5^{\text {th }}$ APSN Plenary Meeting, Naypyidaw, September 2014) <http://apsn-safeguards.org/sites/ default/files/APSN $\% 20 P 1$ enary $\% 202014 \% 20-\%$ 20WG\%20II.pdf>

[5] Y. Naoi, N. Kobayashi, and M. Senzaki, "Activities at the Integrated Support Center for Nuclear Nonproliferation and Nuclear Security, and Trilateral Harmonization among Japan, ROK, and China" (Paper presented to 55th Annual Meeting of the Institute of Nuclear Materials Management, Atlanta, July 2014)
[6] Agreement between the Japan Atomic Energy Institute and the European Atomic Energy Community represented by the Commission of the European Communities in the field of Nuclear Material Safeguards Research and Development, May 28, 1990

[7] Amendment to the Agreement between the Japan Atomic Energy Agency and the European Atomic Energy Community represented by the European Commission in the Field of Nuclear Material Safeguards Research and Development, May 28, 2011

[8] Y. Naoi, "Extension signing ceremony of the European Atomic Energy Community (EURATOM) and the cooperation period involved in the arrangements with the JAEA" ISCN Newsletter 230 (2016) 4-5 (in Japanese)

[9] N. Noro, "Japan's Effort on Promoting Nuclear Security Culture" (Paper presented to 55th Annual Meeting of Institute of Nuclear Materials Management, Atlanta, July 2014) 